\title{
Role of Coronary Prognostic Index, and Thrombolysis, in ST Segment Elevation Myocardial Infarction
}

\author{
Amol Andhale ${ }^{1}$, Anuj Varma ${ }^{2}$, Sourya Acharya ${ }^{3}$, Samarth Shukla ${ }^{4}$, Anuj Chaturvedi ${ }^{5}$, Akhilesh Annadatha ${ }^{6}$ \\ 1,2, 3, 5, 6 Department of Medicine, Datta Meghe Institute of Medical Sciences (Deemed to Be University), \\ Sawangi (Meghe), Wardha, Maharashtra, India, ${ }^{4}$ Department of Pathology, Datta Meghe Institute of \\ Medical Sciences (Deemed to Be University), Sawangi (Meghe), Wardha, Maharashtra, India.
}

\section{ABSTRACT}

Angioplasty is considered superior to fibrinolytic therapy in acute myocardial infarction (AMI) if the patient receives it within the therapeutic window. It is unclear if such advantages are available for patients who need to travel from a community hospital to a facility where invasive care is available, since primary thrombolysis often re-establishes coronary artery blood flow in patients with ST elevation acute myocardial infarction (STEMI).

At the most severe end of the range of acute coronary syndromes is ST segment elevation myocardial infarction (STEMI), which generally occurs when a fibrin-rich thrombus fully occludes an epicardial coronary artery. The diagnosis of STEMI is based on clinical features and persistent ST-segment elevation as evidenced by 12 - lead electrocardiography. Patients with STEMI should have a quick reperfusion treatment evaluation and a reperfusion strategy should be performed immediately following contact with the system.

All patients with AMI who had chest pain within 12 hours were evaluated. The detailed history of chest pain, character, and radiation, had been taken in terms of duration from the beginning of chest pain in minutes. After 10 minutes, patients were given $10 \mathrm{mg}$ of sublingual isosorbide dinitrate and repeated ECG. Patients were excluded if chest pain or ST elevation was resolved after 10 minutes of nitrate administration. In the analysis only those cases in which chest pain and ST shift were not resolved following sublingual nitrates. Serum CKMB estimates have been performed. All patients were treated with 1.5 million IU streptokinase in $100 \mathrm{ml}$ of normal saline for more than 45 minutes. Clinical assessment for 2 hours every half hour was done to evaluate: 1 . Chest pain reduction in a subjective scale percentage and to assess changes in the Killip class. 2. Continuous ECG monitoring of reperfusion rhythm occurrences. Patients are assessed at the end of 2 hours of follow-up for: a. Percentage reduction in subjective chest pain a. A 12 lead ECG to identify changes in the ST height c. Repeat CK-MB estimate. Patients with thrombolysis were classified into two classes on the basis of presence or absence of SCR at the end of two hours of initiation. Those with successful reperfusion were grouped into the SCR Group and into the SCR (negative) Group without successful reperfusion. Coronary prognostic index is a set of questionnaires which prognosticate the outcome in AMI. This review describes the role of Coronary Prognostic Index and thrombolysis in patients of STEMI.

\section{KEY WORDS}

ECG, AMI, STEMI, Angioplasty
Corresponding Author: Dr. Amol Andhale,

Resident,

Department of Medicine. Datta Meghe Institute of Medical

Sciences (Deemed to Be University),

Sawangi (Meghe), Wardha,

Maharashtra, India.

E-mail: dramoljnmc@gmail.com

DOI: $10.14260 / j e m d s / 2021 / 452$

How to Cite This Article:

Andhale A, Verma A, Acharya S, et al. Role of coronary prognostic index, and thrombolysis, in ST segment elevation myocardial infarction. J Evolution Med Dent Sci 2021;10(29):2212-2216, DOI: $10.14260 / \mathrm{jemds} / 2021 / 452$

Submission 18-08-2020,

Peer Review 15-05-2021,

Acceptance 25-05-2021,

Published 19-07-2021.

Copyright (C) 2021 Amol Andhale et al. This is an open access article distributed under Creative Commons Attribution License [Attribution 4.0 International (CC BY 4.0)] 


\section{BACKGROUND}

Coronary artery disease (CAD) is the world's leading cause of death. Moreover, people in the Indian subsoil have one of the highest levels of coronary artery disease in the world. ${ }^{1}$ The disease is often severe, premature in the Indian subcontinent with serious complications and increased youth mortality. It is estimated that in 2004, CAD resulted in 1.46 million deaths, 14 per cent of 10.3 million deaths. ${ }^{2}$ CAD in India is more prevalent in urban areas than in rural India. ${ }^{3}$ This will further increase the burden of CAD on the Indian health system. There is also a need to build effective CAD management strategies in India. Myocardial infarction can be defined from a number of different perspectives related to clinical, electrocardiographic (ECG), biochemical and pathologic characteristics. It is accepted that the term myocardial infarction reflects death of cardiac myocytes caused by prolonged ischaemia. ${ }^{4}$

Thrombolysis for the treatment of acute myocardial infarction is a significant occurrence in the diagnosis of acute myocardial infarction, as the symptoms of acute myocardial infarction have shifted. It has been shown convincingly that early opening of the infarction related artery involves reducing the infarction duration, maintaining left ventricular function, and enhancing clinical outcome.5-8 Since angiography is not available in every hospital, an increasing interest is being shown in simple non-invasive patent tests. ${ }^{7}$ In fact, after thrombolysis, clinical signs in acute myocardial infarction can have a greater functional correlation than an isolated angiographic patent image. ${ }^{8}$

A coronary prognostic index (CPI) has also been constructed in the literature for the prediction of mortality in patients with acute myocardial infarction (AMI). The longterm survival CPI should provide an unbiased tool for testing new types of care for heart diseases in groups of patients in the chronic period. Of the steps that may prolong life after a myocardial infarction, neither long-term anticoagulants ${ }^{9}$ nor reduction of serum cholesterol have been widely popular. ${ }^{10-11}$ More recent therapies include long-term beta - adrenergic blocking drugs, and coronary artery surgery with venous aortic grafts to the right or left coronary artery. For any unbiased trial, it would be important to align the care and control groups of patients according to the seriousness of their illness, and the CPI should provide a valuable means of doing so. ${ }^{12}$ In view of the widening indications for thrombolysis in patients with acute myocardial infarction, new and earlier prognostic indicators are required, which can predict the clinical outcome after thrombolysis \& will help to decide whether the patient should undergo coronary angioplasty. Hence this review was undertaken to review the role of CPI and thrombolysis in MI.

\section{Role of ALT, AST, LDH, CK, and Troponins in Diagnosis of AMI}

In the definition of acute coronary syndrome (ACS), AMI has found its place. ACS comprises a group of clinical syndromes ranging from dysfunctional angina pectoris, AMI with non - S (immediately after ventricular contraction downward deflection) - segment elevation and $\mathrm{T}$ (recovery of ventricles) - segment elevation to AMI, with ST - segment elevation and sudden death. ${ }^{13}$
Electrocardiography (ECG) sensitivity and specificity are poor in ACS. Mostly AMI with a Q wave (downward deflection immediately preceding ventricular contraction) develops in majority of the cases with ST segment elevation in ECG and common ischemic chest pain, and AMI without a $Q$ wave develops in a few. Most non - ST segment elevation cases, however, experience unstable angina pectoris or AMI without a $\mathrm{Q}$ wave, with a few developing $\mathrm{Q}$ wave AMI. When an oxygen free atmosphere occurs, ST segment elevation transforms into ST - segment depression. ${ }^{14}$

Because ECG sensitivity and specificity are poor for diagnosing AMI, the European Society of Cardiology (ESC) and the American College of Cardiology (ACC) have agreed on the requirements for AMI. ${ }^{15}$ Accordingly, patients must have at least two of the following symptoms: normal symptoms, elevation or decrease in cardiac markers (e.g. CK - MB isoenzymes), preferably serum troponins (cTnIor cTnT) Ischemia causes loss of ventricular function and myocardial necrosis due to reduced coronary artery flow. Enzymes such as ALT, AST, LDH, CK, and troponins have, therefore, been markers of AMI diagnosis for years. ${ }^{16}$

Ong et al. ${ }^{17}$ (1983) have reported rapid release of CK - MB in $24(46.15 \%)$ patients while in $53.85 \%$ patients, release of creatine kinase was slow. It was observed that patients with rapid release of $\mathrm{CK}$ - $\mathrm{MB}$ had greater increment in the enzyme level as compared to those with slow release $(P=0.03)$, suggesting enzyme washout.

Garabedian et al. ${ }^{18}$ (1988) also found correlation between early rise of $\mathrm{CK}-\mathrm{MB}$ and successful coronary reperfusion. In 32 patients who underwent thrombolysis in AMI with recombinant tissue plasminogen activator (rt - PA), reperfusion occurred in $70 \%$ patients with left anterior descending artery occlusion and $73 \%$ patients with right coronary artery occlusion. When a greater than or equal to 2.5 times the increase in the CK - MB level was used to identify reperfusion of left anterior descending artery, 14 (70 $\%$ ) out of 20 patients had greater than 2.5 -fold rise. Using this criterion, 13 (93\%) of 14 patients with reperfusion and 5 (83 $\%)$ of 6 patients with persistent occlusion were correctly identified.

\section{Thrombolysis}

Fletcher et al. ${ }^{19}$ reported the first use of thrombolytic therapy in patients with acute myocardial infarction in 1958. In the early 1970s, 24 experiments were performed to determine the efficacy of intravenous streptokinase. According to current standards, both of these studies have significant limitations, so patients were randomized before 72 hours after beginning myocardial infarction. The theoretical basis for thrombolysis was also not clear because debate existed about the cause of AMI intracoronary thrombosis. Along with the lack of proof of streptokinase effectiveness in the trials, further work in this form of therapy has been abandoned.

In 1969 intracoronary streptokinase was administered in Russia by Chazov. ${ }^{20}$ But the interest in thrombolysis was rejuvenated by Rentrop et al. ${ }^{21}$ (1979). For five AMI patients, the influence of both intracoronary nitroglycerine and streptokinase was evaluated. Four out of 5 patients were given intracoronary Streptokinase after a long-term reduction of nitroglycerine at the site of acute occlusion, linked to symptoms alleviation. 
The dosage of streptokinase used was 15 - 60 minutes for 1000 - $2000 \mathrm{rpm}$. Authors concluding that the results of myocardial infarction indicated an underlying factor due to the thrombotic occlusion of coronaries with coronary spasm. Additional work by workers in this area has verified the thrombus as a source of occlusion contributing to AMI.

\section{CRITERIA FOR THROMBOLYSIS IN ACUTE} MYOCARDIAL INFARCTION

Based on ACC / AHA guidelines adapted from a special report by Anderson et al. (1993) ${ }^{22}$ for early management of patients with acute myocardial infarction, the criteria for thrombolysis are -

1. Chest pain consistent with AMI.

2. Electrocardiographic changes - ST segment elevation > $0.1 \mathrm{mV}$ in at least two contiguous leads, new or presumably new bundle branch block.

\section{Time from Onset of Symptoms}

- $\quad<6$ hours - most beneficial.

- 6 - 12 hours - lesser but still important benefits.

- $\quad>12$ hours - diminishing benefits but may still be useful in selected patients.

\author{
Absolute Contraindications \\ 1. Active internal bleeding (excluding menses). \\ 2. Suspected aortic dissection. \\ 3. Recent head trauma or known intracranial neoplasm. \\ 4. History of cerebrovascular accident known to be \\ haemorrhagic. \\ 5. Major surgery or trauma $<2$ weeks.
}

\begin{abstract}
Complications of Thrombolytic Therapy
Complications of thrombolytic agents are the most common and potentially most severe when observed. Most bleedings of all agents are fairly minor. In total, 70 per cent of episodes of bleeding occur at the site of vascular puncture. The most severe of all bleeding complications is intracranial haemorrhage; the incidence varies with the patient's clinical characteristics and thrombolytic agent used. Allergic reactions were reported in $1.7 \%$ of patients in the international Tpa / SK mortality trial. In about $10 \%$ of cases, hypotension occurred.23 Other complications of thrombolytic therapy may include increased risk of myocardial rupture, poor heart defect and cardiac shock, splenic rupture and embolization of cholesterol. ${ }^{24}$ Other problems may be attributed to the use of thrombolytic therapy. ${ }^{25}$
\end{abstract}

\section{Markers of Reperfusion}

The success of coronary re-channelling after thrombolytic treatment can be tested with various reperfusion markers.

1. Invasive marker - e.g. coronary angiography.

2. Non-invasive markers - e.g. resolution of ST elevation on ECG, reduction in chest pain, increase in serum CK, myoglobin and cardiac troponins.
Coronary angiography was considered a gold standard to assess reperfusion success. More than 20 trials were conducted during the $1980 \mathrm{~s}$, which collectively enrolled over 8,000 patients and determined infarction filmed artery (IFA) patentability at 60 minutes, 90 minutes, 180 minutes and one and 21 days after thrombolisation, in various thrombolytic regimes.92 .The majority of investigators focused on infarct related artery (IRA) status at 90 minutes and described TIMI FLOW (Thrombolysis In Myocardial Infarction trials) grading system as

- $\quad$ Grade 0 - Complete occlusion of IRA.

- Grade 1 - Some penetration of contrast material beyond the point of occlusion but without perfusion of distal coronary bed.

- $\quad$ Grade 2 - perfusion of entire infarct related vessel into distal bed but with delayed flow as compared to normal artery.

- $\quad$ Grade 3 - full perfusion of IRA with normal flow. ${ }^{26}$ Thrombolytic therapy aims mainly to restore infarct artery blood flow fast, completed and sustained. The GUSTO 1 angiographic studies (1995) are closely associated to IRA patency with an increased Alteplase mortality rate of 90 minutes. Regardless of the thrombolytic agent used for 90 minutes with an occluded IRA (TIMI grade 0 , or 1 flow) with 8.9 percent thirty-day death rate and "normal" perfusion (TIMI grade 3 flow), with 4.0 percent mortality rate .95 The intermediate mortality rates for patients with "partial" reperfusion (TIMI grade 2 flow) were 7.4 percent.

Moreover, the left ventricular ejection fraction was 5 and 7 days longer, the end systolic volumes smaller and the regional wall movement in infarction zones lower in patients with TIMI Grade 3 flow compared to those with lower TIMI flow rates. The purpose of thrombolytic therapy should therefore be considered as the TIMI grade 3 flow. ${ }^{27}$

Early re-occlusion causes failure of the ventricular function and doubles the mortality rate. However, earlier reocclusion benefits may be offset by subsequent re-occlusion of IRA. Depending on the thrombolytic agent used, the rate of re-occlusion ranges from $4.9 \%$ to $2.5 \%$. Late re-occlusion (which occurs until 1 year after reperfusion) may happen in $25 \%$ to $30 \%$ of IRAs. ${ }^{28,29}$ The IRA 's long term patentability has shown that an independent prediction factor can be associated with a poor result, even silent late re-occlusion.

\section{Limitations of Clinical Markers of Reperfusion} The importance of clinical markers in determining positive reperfusion may be confused with multiple factors. Patients with AMI often need pain control analgesics, which may interfere with assessment of chest pain reduction; similarly, myonecrosis results in spontaneous relief from pain. Chest pain in diabetics that may have painless infarcts can be difficult to determine.

A further ST rise may occur in patients with positive recanalization of AMI during thrombolysis, and some of the staff have used this further ST rise in thrombolysis as a reperfusion marker. ${ }^{30}$

A drop in the ST rate may occur after the AMI, which may present a problem in evaluating it as reperfusion marker. Spontaneous reperfusion arrhythmias may occur during early AMI hours, which reduces their ability to predict successful reperfusion. Evaluation of $\mathrm{CK}-\mathrm{MB}$, myoglobin, and cardiac 
troponins, particularly in patients from the lower socioeconomic population, can be full of financial obstacles. ${ }^{30}$

\section{Role of CPI in Diagnosis of AMI}

But to increase the specificity and sensitivity, new \& earlier prognostic indicators are required, which can predict the clinical outcome after thrombolysis \& will help to decide whether the patient should undergo coronary angioplasty or not. In this direction, the coronary prognostic index (CPI) has also been constructed in the literature for the prediction of mortality in patients with acute myocardial infarction (AMI). The long-term survival CPI provides an impartial tool for testing new types of heart disease care in chronic stage patient groups. No long-term anticoagulants or reduction in serum cholesterol have become widely common for measures that could extend life after a myocardial infarction. For any unbiased trial it will be important to balance treated and control groups of patients according to the seriousness of their disease and CPI should provide useful means of doing this. ${ }^{31-33}$

Age, cardiac enlargement, and the presence of heart failure in the chest x-ray on admission to hospital, are of approximately equal relevance for predicting hospital survival, and three-year survival of the hospital survivors. The next factor, a history of previous infarction, is much more relevant for long term survival than for short-term survival. The next two factors, admission systolic blood pressure, and position of infarct as assessed from the 12 lead ECG, were more relevant to short term than to long-term survival, and consequently they were not included in the long-term index. Other factors such as past history of hypertension or diabetes, or the presence of obesity, were not clearly relevant either to short-term or long-term survival, and therefore they were not included in the index. To summarize, the factors included in the coronary prognostic index for short term survival are age, cardiac enlargement, the presence of left ventricular failure (both assessed from the chest, x-ray), a history of previous myocardial infarction, a low systolic blood pressure, and the position of infarct on the ECG, (anterior infarction being more serious). The factors were the same for long-term survival, except that admission systolic blood pressure and position of infarction were not included. ${ }^{31,32}$

R. M. Norris et al. ${ }^{33}$ analysed coronary prognostic index for predicting survival after recovery from acute myocardial infarction. A three-year follow-up was recorded for 530 patients who had left the hospital following an acute myocardial infarction. To evaluate as to which easily observable clinical characteristics were correlated with acute mortality in hospital, these were the survivors from a group of 757 cases examined. The aim of the follow-up was to decide which variables predicted survivors' three - year mortality from the acute attack and to formulate a coronary prognostic index (CPI) that would predict survival for three years. Of the factors investigated, only four were important to three - year survival, i.e., age, heart size, degree of pulmonary congestion, and prior ischaemia. By using the CPI for three year survival based on these variables, patients may be divided into five classes with a three - year mortality varying from $12 \%$ to $85 \%$. In evaluating the effects of care in groups of patients who have recovered from acute myocardial infarction, the CPI for three - year survival should be of importance.
Woo KS 34 in 1987 assessed coronary prognostic index for the Chinese. With data from 644 Chinese patients in Hong Kong, a coronary prognostic index (CPI) for the prediction of mortality of patients with acute myocardial infarction (AMI) has been constructed. According to this CPI, according to their age, blood pressure, heart size, serum urea level, positions and types of infarctions, the occurrence of pulmonary congestion and cardiac dysrhythmia in the initial three days, patients with AMI may be divided into subgroups with growing hospital mortality from $1.6 \%$ to $100 \%$. This basic and convenient CPI could be useful both in China and in many other developing countries in the Asia - Pacific region for the objective assessment and stratification of AMI.

\section{CONCLUSIONS}

This simple and convenient CPI could be useful for the objective assessment and stratification of AMI in the Indian population. The plain, realistic, and economic design of this index will also be very attractive in many other Asia - Pacific countries for the evaluation of AMI patients. These findings indicate that the coronary prognostic index has a greater capacity for detecting reperfusion following thrombolytic treatment than the other markers tested.

Financial or other competing interests: None.

Disclosure forms provided by the authors are available with the full text of this article at jemds.com.

\section{REFERENCES}

[1] Enas EA, Yusuf S, Mehta JL. Prevalence of coronary artery disease in Asian Indians. Am J Cardiol 1992;70(9):945-9.

[2] Huffman MD, Prabhakaran D, Osmond C, et al. Incidence of cardiovascular risk factors in an Indian urban cohort results from the New Delhi birth cohort. J Am Coll Cardiol 2011;57(17):1765-74.

[3] Chadha SL, Gopinath N, Shekhawat S. Urban-rural differences in the prevalence of coronary heart disease and it's risk factors in Delhi. Bull World Health Organ 1997;75(1):31-8.

[4] Myocardial infarction redefined--a consensus document of the Joint European Society of Cardiology/American College of Cardiology Committee for the redefinition of myocardial infarction. Eur Heart J 2000;21(18):1502-13.

[5] Christian TF, Schwartz RS, Gibbons RJ. Determination of infarct size in reperfusion therapy for acute myocardial infarction. Circulation 1992;86(1):81-90.

[6] White HD, Cross DB, Elliot JM, et al. Long term prognostic importance of patency of infarct related coronary artery after thrombolytic therapy for acute myocardial infarction. Circulation 1994;89(1):61-7.

[7] GUSTO Angiographic Investigators. The effect of tissue plasminogen activator, streptokinase or both on coronary artery patency, ventricular function and survival after acute myocardial infarction. $\mathrm{N}$ Eng J Med 1993;329(22):1615-22.

[8] Brugada P, Waldwacker B, Kersschof Y, et al. Ventricular arrhythmias initiated by programmed stimulation in 
four groups of patients with healed myocardial infarction. J Am Coll Cardiol 1986;8(5):1035-40.

[9] Second Report of the Working Party on Anticoagulant Therapy in Coronary Thrombosis to the Medical Research Council. An assessment of long-term anticoagulant administration after cardiac infarction. $\mathrm{Br}$ Med J 1964;2:837-43.

[10] Leren P. The effect of plasma cholesterol lowering diet in male survivors of myocardial infarction. A controlled clinical trial. Acta Med Scand Suppl 1966;466:1-92.

[11] Report of a Research Committee to the Medical Research Council. Controlled trial of soya-bean oil in myocardial infarction. Lancet 1968;2(7520):693-9.

[12] Norris RM, Caughey DE, Deeming LW, et al. Coronary prognostic index for predicting survival after recovery from acute myocardial infarction. Lancet 1970;2(7671):485-7.

[13] Cervellin G, Rastelli G. The clinics of acute coronary syndrome. Ann Transl Med 2016;4(10):191.

[14] Dizon JM, Brener SJ, Maehara A, et al. Relationship between ST-segment resolution and anterior infarct size after primary percutaneous coronary intervention: analysis from the INFUSE-AMI trial. Eur Heart J Acute Cardiovasc Care 2014;3(1):78-83.

[15] Nagurney JT, Huang C, Heredia O, et al. The new and old definitions of acute myocardial infarction: a data-based comparison. Am J Emerg Med 2008;26(5):523-31.

[16] Martin TN, Groenning BA, Murray HM, et al. ST-segment deviation analysis of the admission 12-lead electrocardiogram as an aid to early diagnosis of acute myocardial infarction with a cardiac magnetic resonance imaging gold standard. J Am Coll Cardiol 2007;50(11):1021-8.

[17] Ong L, Reiser P, Coromilas J, et al. left ventricular function and rapid release of creatine kinase in acute myocardial infarction: evidence for spontaneous reperfusion. N Eng J Med 1983;309(1):1-6.

[18] Garabedian HD, Gold HK, Yasuda T, et al. Detection of coronary artery reperfusion with creatine kinase-MB determination using thrombolytic therapy: correlation with acute angiography. J Am Coll Cardiol 1988;11(4):729-34.

[19] Fletcher AP, Alkjaersig N, Smyrniotis FE, et al. The treatment of patients suffering from early myocardial infarction with massive and prolonged streptokinase therapy. Trans Assoc Am Physicians 1958;71:287-96.

[20] Chazov El, Matveeva LS, Mazaev AV, et al. Intracoronary administration of fibrinilysin in acute myocardial infarct. Ter Arkh 1976;48(4):8-19.
[21] Rentrop KP, Blanke H, Karsch KR, et al. Acute myocardial infarction: intracoronary application of nitroglycerin and streptokinase. Clin Cardiol 1979;2(5):354-63.

[22] Anderson HV, Willerson JT. Thrombolysis in acute myocardial infarction. N Eng J Med 1993;329(10):703-9.

[23] A randomized trial of intravenous heparin versus recombinant hirudin for acute coronary syndromes. The global use of strategies to open occluded coronary arteries (GUSTO) IIA investigators. Circulation 1994;90(4):1631-7.

[24] Randomized trial of intravenous streptokinase, oral aspirin, both or neither among 17,187 cases of suspected acute myocardial infarction: ISIS-2. ISIS-2 (Second International Study of Infarct Survival) collaborative group. Lancet 1988;2(8607):349-60.

[25] Queen M, Biem J, Moe GW. Development of cholesterol embolization syndrome after intrvenous streptokinase for acute myocardial infarction. Am J Cardiol 1990;65(15):1042-3.

[26] TIMI Study Group. The Thrombolysis in Myocardial Infarction (TIMI) trial. Phase I findings. N Eng J Med 1985;312(14):932-6.

[27] White HD, Frans JJ, Van De Werf. Thrombolysis for acute myocardial infarction. Circulation 1998;97(16):1632-46.

[28] Meijer A, Verheugt FW, Werter CJ, et al. Aspirin versus coumadin in the prevention of reocclusion and recurrent ischemia after successful thrombolysis: a prospective placebo-controlled angiographic study: results of APRICOT Study. Circulation 1993;87(5):1524-30.

[29] White HD, French JK, Hamer JW, et al. Frequent reocclusion of patent infarct-related arteries between 4 weeks and 1 year: effects of antiplatelet therapy. J Am Coll Cardiol 1995;25(1):218-23.

[30] Kircher BJ, Topol EJ, O'Neil WN, et al. Prediction of infarct related artery recanalization after intravenous thrombolytic therapy. Am J Cardiol 1987;59(6):513-5.

[31] Norris RM. Fatality outside hospital from acute coronary events in three British districts, 1994-5. United Kingdom Heart Attack Study Collaborative Group. BMJ 1998;316(7137):1065-70.

[32] Norris RM, Caughey DE, Mercer CJ, et al. Prognosis after myocardial infarction. Six-year follow-up. Br Heart J 1974;36(8):786-90.

[33] Norris RM, Caughey DE, Deeming LW, et al. Prognosis following acute myocardial infarction. $\mathrm{N}$ Z Med J 1973;77(488):12-8.

[34] Woo KS. Coronary prognostic index for the Chinese. Aust N Z J Med 1987;17(6):562-7. 